\section{A COURSE OF}

\section{LECTURES ON SURGERY,}

\author{
DELIVRRED IN THB
}

MEDICAL SCHOOL OF CAMBRIDGE.

By GEORGE MURRAY HUMPHRY, Esa., Dowìing College, Surgeon to Addenbrooke's Hospital.

\section{Lecture XXXII.}

Scirrhous and Encephaloid Cancer.

Characters of scirrhous tumours; certain growths in their minute structure resemble fibrous tumour, but present the general characters of cancer, and should therefore be regarded as scirrhous; scirrhus of intestinal mucous coat an example of this; tendency of tissues affected with scirrhus to contract; the term "scirrhus" should be applied only to induration of a cancerous nature; diagnosis of scirrhus from inflammatory induration in the breast, in the intestinal canal; ages, persons, and parts most liable to be affected by it.

Characters of encephaloid tumours; extravasations of blood in them; presence of cartilage in them; they are occasionally invested with capsules; they become "fungous;" persons and parts most liable to be affected with them; osteo-encephaloma; difficulties in the diagnosis of encephaloid disease.

Having entered somewhat at length into the subject of malignant disease generally, and of the epithelial variety of cancer, I shall not detain you much with the other varieties that have been enumerated, because you have abundant opportunities of clinical instruction with regard to the most important of them, and because $I$ have little information to communicate, which is not contained in the ordinary treatises upon this subject.

The "scirrhous" and " encephaloid" or hard and soft varieties of cancer present 80 many points of correspondence in their general features, that it seems better to consider them under one head. Both consist of cells and molecules contained in the areolie of a fibrous stroma; the chief difference depending upon the relative proportion which these constifuents bear to one another. The cellular element predominating in the encephaloid, the fibrous element in the scirrhous cancer. The well-marked examples of each are sufficiently distinct to the eye and the touch, but the interval between these is filled up by a closely-connectied series of specimens, many of which we are unable to refer to either species. Moreover, it not uncommonly happens that we find cancer in different organs of the same patient, which, approaches both to the seirrhous and to the encephaloid type; or it may be, that in the same cancerous tumour one portion resembles encephaloid whilst another is more nearly allied to seirrhas.

A "scirhors" tumour is usually a hard, beaviv, nodulated mass, blended with the surrounding tisbues, which ran into and are lost in it; or they mey be con- densed and adherent to it. It grates under the knife; and its section has a pale, glistening, or dull white colour. Very often it is marked with streaks or bands more opaque than the rest of the mass, interlacing together and dispersed without any regulcr arrangement. Sometimes a numbe of yellowish white soft spots are scattered through it; or there may be small cavities containing a purulent fluid. A certain amount of fluid oozes, or may be compressed, from its cut surface. When the tumour is very hard this is scanty, thin, and pale, like serum; if the tumour be softer, the expressed fluid is more abundant and has a creamy appearance. In either case it contains cells, nuclei, and granules, with the addition, often, of corpuscles. The distinctness with which the fibres or bands are visible to the naked eye varies a good deal. In some instances they cannot be seen at all, the mass presenting an uniform, hard, brawny, cartilage-like appearance ; or it may be composed of pale glistening grains closely compressed together, without any show of interlacing bands and fibres. In other cases, more particularly in cancer of the liver, you may see a reticulum of coarse, thick, tough bands, radiating, perhaps, from the centre to the circumference, like the spokes of a wheel, or disposed without any definite arrangement, and including in their meshes a soft, yellowish, or creamy fluid.

When a thin section is examined under the microscope, it is found to consist of a meshwork of fibrous tissue, forming wavy bands, which are sometimes arranged in circles, but generally are disposed without any apparent order. The circles or meshes enclose a fluid containing nucleated corpuscles and granules. The whole structure is rendered more transparent by acetic acid, which has the effect of partially or completely dissolving the walls of the corpuscles, so rendering the nuclei more visible. In its minute structure, therefore, a scirrhous tumour differs from a fibrous, mainly if not entirely, in the presence of cancer cells, infiltrating between its component fibres. I do not mean to say that it is the only real difference between the two growths, because, as has been already stated, it is prebable that the malignant qualities-the tendency that is to ulcerate and spread-are not confined to the cells alone, but they are shared, though in a far less degree, by the fibrous tissue also. This is an important point to bear in mind in connection with the fact that the cell-element varies very greatly in its relative proportion to the fibrous element in different specimens of scirrhus. In some instances the cancer cells can with difficulty be distinguished from the developmental cells of the tissues, and in some there is reason to suppose that they are absent altogether, although the tumour may still present the genuine qualities of cancer. It is important, I say, to remember that the malignant qualities of cancer are shared by the fibrous tissue, as well as by the cells entering into its composition, because it seems to afford an explanation of those cases, where tumours supposed to be fibrous, and presenting to experienced observers the anatomical, microscopical, and chemical characters of fibrous tumours, have returned after complete extirpation, have proceeded to sloughing and ulceration like 
ordinary cancerous growths, and have infected distant organs. Specimens of tumours which were regarded to be fibrous, and which took the course just mentioned, are described in the catalogue of the Museum of the College of Surgeons. One of the patients, whose case is there related, I had the opportunity of examining before the operation was performed, and the general characters of the growth certainly appeared to me to betoken scirrhous rather than fibrous disease. I should imagine that some of the cases described by Dr. Bennett under the title of " fibro-nucleated cancroid growth," are of the kind of which I am now speaking, being composed of filaments infiltrated with oval nuclei, constituting a densely fibrous texture, which grated under the knife. Yet these tumours grew quickly, involved the adjacent textures, were liable to softening and disintegration, and, if excised, returned at the place originally affected. They presented, in short, the usual general characters of scirrhus, though in their microscopical structure they were more nearly allied to fibrous growths. In referring such cases to the pathological division which they should occupy, and in ranging them among simple or malignant growths, we must rely either upon the general or the microscopical characters which they present. The reasons for preferring the former have been given in a preceding lecture; and it certainly does seem the best practical arrangement to include in th: same class those tumours which present the great weneral features of cancer, although they may not quite agree in their minute structure.

I have already called your attention to another instance of the same kind, viz., the thickened, indurated, condition of the muscular coat of the intestinal canal, which accompanies scirrhus. The absence, nearly complete, of cancer-cells in the tunic thus affected, has caused great doubt as to the real nature of the disease. By some pathologists it has been thought to depend merely upon hypertrophy; by others it is regarded as an inflammatory induration. The peculiar stony character of the induration, sufficient to cause a grating under the knife; its usual association with cancer of the mucous membrane of the adjacent absorbent glands and of the other parts of the body; the period of life and circumstances under which it occurs; its liability to softening, ulceration, \&c., are characters so well marked, and so clearly attaching the disease to scirrhus, as to leave no doubt of its real nature upon my mind. I may mention that the position which this scirrhous induration is found to occupy, is a point of much practical importance, enabling us in many instances, to distinguish during life those strictures of the alimentary canal which are sure to prove fatal from those which admit of alleviation by treatment. Thus the simple or inflammatory strictures are found, as has been mentioned in a former lecture, exclusively, or almost exclusively, near the terminations of the canal, that is to say, at the upper part of the œesophagus, and at the lower part of the rectum. The malignant strictures, it is true, occur ut these parts, but they are not confined to them. They are often found at the lower part of the œsophagus, in the stomach, very often at the pylorus, sometimes in the upper part of the rectum. Here is a specimen of scirrhous disease of the muscular coat at the sigmoid flexure of the colon. You observe the thickened, indurated, striated condition of the tunic, and the narrowing of the intestine at the part; the submucous tissue is free, and the mucous membrane preserving nearly a healthy condition, is puckered into longitudinal folds, where it passes through the stricture; the neighbouring absorbent glands are enlarged and greatly indurated. The specimen was taken from a woman who had passed the middle period of life, and who died of the disease. Here is a specimen of the same disease affecting the terminal part of the ilium and the ilio-cœcal valve in very much the same way, except that the submucous tissue is involved, and the mucous membrane is in consequence adherent to the thickened and indurated muscular tunic. In this third specimen, taken from a man aged 55, the disease, situated at the junction of the ascending with the transverse portion of the colon, has advanced farther; the mucous membrane being itself involved and destroyed, and a considerable ulcer, with raised everted edges produced; the muscular coat is also in part destroyed. Constriction of the small intestine between the duodenum and the termination of the ilium from either inflammatory or scirrhous induration of its tunics is, I believe, very rare.

A remarkable and important feature connected with the scirrhous conversion of tissues is the tendency of the affected part to contract, much in the same way as cicatrix or recently effused lymph. It is this which gives rise to narrowing of the bowel, or stricture, when the muscular coat is diseased, after the manner seen in the specimens just shown. The same contractile tendency causes the retraction of the nipple when the ducts of the mammary gland are becoming converted into scirrhus, and the close adhesion of the integuments to the breast, when the bands of fibrous tissue passing from one to the other are involved in the same way. This, no doubt, contributes also much to the hardness of scirrhus, and explains many of the phenomena observed in organs affected by it.

Much confusion has arisen, and many erroneous notions have been occasioned, by the vague indefinite manner in which the term "scirrhus" has been used, which, it must be confessed, is warranted by the literal meaning of the word; for it signifies merely, hardness or induration, and is often used to express that only. Most accurate pathologists of the present day, however, employ the term to indicate the variety of hard cancer which we are considering, and it is, therefore, obviously most desirable that the word should be used in that sense strictly, that it should not indicate merely induration, but, in an especial manner, induration of a cancerons nature.

The diagnosis of scirrhous affections, in an early stage, from the swellings, indurations, and contractions, resulting from simple inflammation, is of great practical importance, and, in many instances, of considerable difficulty. You are aware that scirrhus very frequently attacks the mammary gland, and on this account females are, with good reason, suspicious of any swelling 
or induration, or, indeed, of any complaint affecting that organ; and you may expect very often to be called upon to give an opinion respecting diseases of the breast, and will be particularly and anxiously questioned whether the disease is, or is likely to become, cancerous. In the greater number of cases it is easy enough to give a decided and correct answer to the question; but there are many instances in which it is very difficult, if not impossible, to do so. Patients, whose age ranges from 20 to 50, are frequently presenting themselves at the hospital with a lump in the breast, which is most commonly situated on the onter side of the nipple. The lymph is clearly distinguishable by the touch, and gives to the finger the impression that it consists of slight enlargement, with induration and cohesion of the mammary globules; the skin over it may or may not be adherent, and slightly discoloured, and the nipple may or may not be drawn a little towards the part. The part is rather tender, and is usually painful, the pain being very often represented of a pricking or darting character. The patient states that the lump has existed for two, three, four, or more months, and that it gradually increases, and becomes more painful. Now these are the symptoms which commonly attend chronic inflammation of a part of the breast, and this is the way in which scirrhus usually begins. Many circumstances, it is true, assist us to discriminate between the two diseases, such as the age and general appearance of the patient. The inflammatory affection is common at every period in the interval $I$ have mentioned, and, though very generally associated with some disorder of the digestive or uterine function, is quite compatible with soundness of health. Scirrhus occurs most commonly, though not exclusively, towards the decline of life, and is usually associated with some eridences of failure in the nutritive forces, such as wasting or weakness, sallowness of complexion, change of colour in the hair, \&c. Moreover, the scirrhons lump is, for the most part, better defined, and more stony than the inflammatory, and it is often attended with enlargement and induration of the absorbent glands. Nevertheless, errors are continually being committed by the ablest surgeons in attempts to make a diagnosis between these two affections, which should induce you to study both diseases attentively, and to weigh the symptoms well in each case before you give a decided opinion.

It is also highly important to distinguish between the inflammatory and scirrhous strictures of the intestinal tube. The former, as you have just been told, are confined, or nearly confinied, to the upper and lower extremities of the tube, to the commencement of the œsophagus, and the termination of the rectum. In the œsophagus they are of so very rare occurrence that when stricture is situated in any part of that tube the chances are greatly in favour of its being malignant. It is out of the reach of the finger; and $I$ cannot tell any symptoms that would, with certainty, enable us to decide that the disease is of simple nature. In the lower part of the rectum, the disease being within reach of the finger, is more readily diagnosed. Both simple and malignant strictures usually begin about the upper edge of the internal sphincter; and difficulty of defecation, with uneasiness about the parts, especially about the sacrum, are the prominent symptoms in both. The former are most common in young women-so far as my experience goes, in young women who have been the subjects of syphilis. They are attended with muco-purulent discharge, and frequently accompanied by abscesses, induration, or other signs of inflammation about the external parts and the adjacent mucous membrane. The finger finds the stricture, which is often very tight and sharp, to consist in an induration and contraction of the mucous membrane itself, and of the submucous cellular tissue. The stricture dependent upon scirrhous disease is most frequently met with in men who are above forty; is attended with frequent desire to go to stool, and with discharges of blood. There is nothing abnormal in the external parts or in the adjacent mucous membrane. The finger discovers a hard mass, of greater or less size, encircling the bowel and narrowing its calibre; and the mucous membrane may be traced unaltered through the centre of the mass, being puckered into longitudinal folds by the contraction that has taken place on its exterior. The mass just mentioned has close connections with the surrounding parts, rendering it, to a greater or less extent, immoveable. It is a point worth remembering, and which you will find as a general rule to be correct, that strictures depending upon inflammation are caused by induration and contraction of the mucous membrane and submucous tissue; whereas, the scirrhous stricture consists in disease and contraction of the muscular coat, the mucous and submucous layers being in the first instance intact. True, they become involved after a time, and cancer may even commence in them; but it does not then produce so much narrowing of the bowel.

Scirrhus is found to affect most frequently those who are in the decline of life, females about the period of the cessation of the catamenia, and persons whose nutritive energies have been lowered by fatigue, anxiety, or other depressing influence. It may appear, however, in much younger persons, and has even been observed in the fotus in a few instances. It affects, 'so far as I know, individuals of all classes and all temperaments in an equal degree; the light and the dark, the fat and the lean, the plethoric and the anæmic, being alike subject to its ravages, or nearly so. Perhaps persons who are pale, thin, and of dark complexion, are somewhat more predisposed to it than others; but I am not sure of this. Very few organs and tissues are free from its invasions. It is most frequently met with in the mammary gland and the os uteri. It rarely commences in any other part of the uterus bat the os. I have examined many patients, before and after death, and the specimens in several museums, without seeing a well-marked exception to this rule, which is of much practical importance; for if we find the os uteri healthy in a suspected case we may infer, with tolerable certainty, that the whole organ is free from scirrhus. In one case that proved fatal in the hospital the disease affected the middle part of the vagina, leaving the uterus free. It 
sometimes commences in the absorbent glands. It does not usually affect the bones or voluntary muscles as a primary disease, though it often extends to them from other parts, or attacks them secondarily. In this specimen is a considerable scirrhous mass occupying the septum between the ventricles of the heart. The tumour projects into the left ventricle, raising one division of the mitral valve. The patient, (a woman,) from whom this was taken died of scirrhus of the œesophagus in the hospital. No suspicions of cardiac disease were entertained during her life.

The " encephaloid," or softer vaviety of cancer, differs from scirrhus, as has been already said, chiefly in the greater proportion which the cancer-cells and nuclei bear to the fibrous element. These cells appear to be the most active agents in the morbid structure; and it is doubtless in consequence of the large number of them contained in it that encephaloid cancer is so rapid in its progress, as compared with scirrhus. It is also owing probably to the active reproductive properties of these cells that encephaloid tumours attain so great size and are so vascular; the vessels in the adjacent parts being enlarged in a proportionate manner.

The appearances presented by the encephaloid structure vary a good deal in different cases, and in different stages of its progress. Under all conditions it is soft and pulpy, and, when covered by other tissues, it communicates to the finger a sense of elasticity very likely to be mistaken for fluctuation. It may easily be broken down by a probe or by the finger. Commonly it has, when in an early stage, a pale, gelatinous appearance, with more or less of a cream colour, and a shade of pink. Diffused through it at various parts, are probably spots or patches of an opaque vellow colour; these spots are blended with the rest of its substance, at least they are not separated from it by a very welldefined line. Frequently the whole mass has a mere dull, opaque-white colour, resembling not a little the white substance of the brain; and an abundance of thick, creamy fluid oozes from the whole of its cut surface, if slight pressure be made. This fluid often constitutes a large proportion of the substance of the tumour, and escapes from its surface if it be soaked in water for a time, leaving merely a fine reticulated structure, composed of delicate filamentous threads. The appearance so produced you may often see in the moist specimens of soft cancer preserved in museums.

So soft and delicate is the structure of an encephaloid tumour, that the vessels dispersed through it, (which are commonly considerable both in number and size, and, owing to the rapidity of their growth, are not furnished with very stout walls, or great contractile power, resembling much the vessels in granulations), are very liable to give way. Hence the substance of the tumour is often sprinkled with small bloody extravasations; or blood may be infiltrated into its structure, giving it a deep crimson or red colour; and various shades of pink, saffron, and orange will result from changes in the blood thus extravasated. Now and then the effusion of blood takes place in such large quantities and with such force, that the substance of the tumour becomes almoat entirely broken down; or cutting into it you find it to consist of a sac filled with large coagula, with perhsps scarcely sufficient of the proper structure remaining here and there to enable you to decide the real nature of the disease. This tumour, for instance, connected with the femur, is composed of a large hemispherical sac, which was full of black fluid, and softly clotted blood; we might have been at a loss to account for such a circumstance had there not been on one side of the sac a mass of firm, white, medullary matter. The large tumour of the fore-arm here shown, is a still more remarkable instance of the same kind. When recent it was found to consist of a large sac filled with coaugulated blood, of which the greater part looked as if it had been recently effused and coagulated, though some of it near the circumference had probably been longer in the sac. The walls of the sac were lined by a thin layer of soft reddish substance, like lymph, which was without difficulty scraped off; and when it had been removed they were seen to be composed of the adjacent muscular fibres, tendons, and other tissues, stretched, but in a perfectly healthy state. In a few places we found, upon careful examination, on the interior of the sac, small button-shaped masses, which were composed of blood, intermixed with a soft white substance; and at the back of the tumour was a small mass presenting the ordinary appearance of medullary substance without any intermixture of blood. These would all have been overlooked had we not spent some time in examining and dissecting the tumour. The radial and ulnar vessels passed over the sac in close contact with it; they were quite healthy. The limb was removed by amputation from a man, aged 30, who said that a swelling had formed above the wrist three years previously, in consequence of a sprain; for two years it remained nearly stationary ; in the last year it had increased very quickly and been attended with a good deal of pain. The forced flexion of the fingers, caused by the stretching of the flexor muscles, pressed the nails against the lower part of the tumour, and had caused a small ulcerated opening into the sac, through which large quantities of blood had escaped. The tumour measured twenty-two inches in circumference. The man recovered from the operation, and was in good health a year and half afterwards.

Sometimes we find the tumour composed of cysts of various sizes containing serum, or turbid bloody fluid, or coagula, or softened medullary substance, mixed with coagula. The walls of the cysts are thick, and together with the interspaces between them, are infiltrated with medullary substance. The cystic variety and medullary disease is most frequent in the ovary ; but it occurs also in the interior of the bones, and occasionally in other parts of the body.

An interesting and remarkable circumstance to which I have already directed your attention when speaking of enchondromatous tumours, is the occasional presence of nodules of cartilage in the midst of encephaloid growths. They are composed of structure resembling genuine fotal cartilage; and they are imbedded, sometimes in the medullary mass itself, at others in fibrous 
tissue which separates it from the medullary substance. This combination of cartilage with encephaloia structure is met with most frequently in the testicle. It is not quite certain in cases of this kind whether the encephaloid growth is engrafted upon one of a cartilaginous nature; or whether, on the other hand, the encephaloid disease has the priority, and some of its structures undergo a cartilaginous transformation; or, lastly, whether the two diseases-the encephaloid and the enchondromatous-originate and proceed together.

What has been already said of the mode of growth of cancerous tumours and their influence upon the surrounding tissues, applies to the encephaloid variety, no less than to the others. The new material seems to be infiltrated among, or blended with, the natural structures, of which some are hypertrophied, and others disappear or are transformed. The tumour spreads by invading, assimilating, and destroying the surrounding parts, and is, therefore, as a general rule, devoid of any capsule. Sometimes, however, the rate of growth of the interior of the mass exceeds considerably the rate at which it involves the contiguous structures; in such case it attains a great size, displaces the adjacent parts, stretches and compresses the cellular tissue, and forms a capsule for itself after the manner of a simple tumour. Thus it is in consequence of the greater rapidity of their growth that encephaloid tumours are more frequently capsulated than scirrhous. The presence of a capsule investing and isolating them from surrounding parts of course greatly facilitates their removal by operation.

Probably nothing has so great an influence in restraining the growth of these tumours as pressure. Its effect in this way may be estimated, by observing the rapidity with which the morbid product sprouts forth when the skin and other tissues which had covered the tumour give way, and are destroyed by ulceration. It grows very luxuriantly at the exposed part, and soon forms a considerable mass, like a fungus, overhanging the surrounding skin. The occasional manifestation in this way of that property of rapid growth which forms an important feature in the encephaloid group of cancer, has caused the term "fungous" or "fungoid" to be applied to it in all its stages. The word " hæmatodes" ("fungus hæmatodes") superadded by Hey and other surgeons, is expressive of the liability of these fungous growths to bleed frequently, and freely.

Soft cancer is more frequently found at an early period of life than scirrhus, being met with in the foetus, and at every period after birth. Children with darkbrown hair, and florid rosy complexion, are not liable to it; but $I$ have seen it attack the pale, light-haired, and leuco-phlegmatic adults, and old people are by no means exempt from its visitations, though the chance of being afflicted by it decreases as they advance in years. Like scirrhus, it affects a great variety of organs, but is most frequently found in those parts which are least subject to scirrhus, viz., the testicle, the ovary, the eye, and the fundus of the uterus. Sometimes it begins at the os uteri. It often attacks the exterior of the bones, more particularly the superior maxillary bones, the pelvis, and the femur. Under such circum- stances the medullary matter is infiltrated between the bone and the fibres of the periosteum, the latter becoming enlarged, thickened, and ossified, from the spirules, layers, and masses of new osseous matter which are so commonly found shooting from the bone into the substance of the tumour. Enough has been said upon this in a former lecture, and I would now only remind you of the remarks that were then made, and exhibit to you this specimen which has been lately obtained; it was taken from a lad, aged 13, who had for five years complained of aching pain about the right thigh. When we first saw him, two years before death, there was a hard swelling around, and evidently connected with, the upper part of the shaft of the femur. It felt like a mere thickening of the bone, but was larger than a swelling from that cause usually becomes in so young a person; the muscles moved freely over it, and we supposed it to be some tumour growing from the femur. Such tumours at that time of life, in that situation, being for the most part of encephaloid character, we gave an unfavourable opinion, notwithstanding the extreme hardness of the mass, which might have been thought incompatible with the existence of soft cancer. The disease progressed slowly for some time, but in the last year its advances were more rapid, and the tumour became less hard, and assumed more the appearance of an encephaloid growth. You see that the tumour is composed of two distinct structures-a central solid mass of very dense heavy bone, and a thick circumferential layer of pale, soft, medullary substance. The bony mass is two or three times as thick as the femur, whose place it occupies, and it presents no trace of medullary cavity or other rudiments of the femur. A magnifying glass shows it to consist of a dense network of thick osseons fibres, including very small areolæ, and a fine section exhibits, under the microscope, tolerably distinct haversian canals and corpuscles, somewhat less regular, but sufficiently resembling those of true bone. The encephaloid substance affords numberless large compound cancer-cells. The two structures are in close contact, and united by continuity of tissue, though their line of junction is well defined. The lower part of the shaft of the femur can be seen gradually expanding into the tumour, and surrounded by medullary and bony matter, the two structures being here blended together. It is the most remarkable instance of the tendency to hypertrophy or hyperostosis, as it is called, in bones affected with encephaloid disease that I have met with. A few years ago the term "osteo-sarcoma" used to be generally applied to tumours of this kind. Perhaps "osteoencephaloma" would be better, as conveying a more accurate idea of the real nature of the disease. Moreover, the word "sarcoma" being of very indefinite signification, is, in the present day, employed by few accurate pathologists. Most, if not all, the tumours described by Müller under the term " osteoid," are, I believe, examples of this kind, or, more rarely, of simple tumours in course of ossification. Sometimes, as in this specimen of encephaloid disease of the femur, the medullary substance is infiltrated into the cancellous 
tissue of the bone, and between the layers which form its walls, rending the latter asumder, and expanding them to considerable thickness. Here, too, the cancellous tissue at one part of the femur is solid, very hard, "ivory-like," presenting precisely the appearance described to belong to osteoids. When the disease originates in the cancellous texture of the interior of the bones, it is generally in the articular extremities, rarely in the medullary tubes of their shafts ; and it is remarkable how long the articular cartilages resist the ravages of the disease, and preserve the immunity of the joint long after the adjacent part of the bone has been entirely destroyed.

Soft cancer sometimes attacks the muscles primarily, and now and then it makes its appearance in the skin. When it does so the tissue of the cutis and of the subcutaneous areolar membrane, become infiltrated with the morbid product, and the surface of the skin may be raised into little rounded tumours, somewhat resembling those occurring in molluscum. Hence the term "mollusciform" cancer has been applied to the disease presenting this form.

The difficulties in the diagnosis of soft cancer are practically very many and very great. I can but warn you of them, for experience and caution only can prevent your being occasionally deceived. Probably I am correct in saying that no one other disease has been the source of so many capital blunders in the practice of surgery. In the first place, the age of the patient, his healthy florid complexion, and well-nourished frame, often throw us off our guard, and prevent the suspicion of so desperate a disease. Secondly, the elasticity of the tumour, which the nicest touch may be unable to discriminate from fluctuation, together with its quick formation, the vascularity, tenderness, and perhaps œdema of the superficial tissues, suggest the probability of an abscess; but blood instead of pus follows the incision, and a probe or the finger passes into a soft brain-like mass, small portions of which probably escape, and present the general and microscopical characters of medullary cancer. The same features that cause encephaloid tumour to be mistaken for an abscess, or some of them, are likely to cause it to be mistaken for a cyst or a fatty growth. I have already mentioned an instance of this kind, in which an operation, commenced for the purpose of removing a fatty tumour, was suspended in consequence of the hæmorrhage that ensued, and the discovery that the disease was of malignant nature. Thirdly, these encephaloid tumours, owing to the number and size of the vessels distributed to them, are occasionally found to be attended with pulsation, and have, for that reason, been, in several instances, mistaken for aneurism, and treated by ligature of the neighbouring large vessels. Thus the femoral artery has been taken for a pulsating tumour on the inside of the thigh, the internal iliac for a pulsating tumour of the buttock, and the common iliac for a pulsating tumour of the iliac region; and in each of these cases the disease proved to be malignant, In all, doubtless, the utmost attention was given to ensure a correct diagnosis ; and in each we may unhesi- tatingly believe that the mistake depended upon the complexity of the symptoms rather than upon any want of tact or caution on the part of the surgeons. There are many other sources of fallacy connected with the diagnosis of soft cancer. I have in two instances known amputation performed for malignant disease of the internal condyle of the femur, by surgeons of eminence, under the impression that the affection was only the common synovial disease of the knee-joint.

\section{ON THE PROGRESS}

or

\section{PUBLIC HYGIẼNE AND SANITARY LEGISLATION IN ENGLAND,}

\section{AND THE ADVANTAGES TO BE DERIVED FROM THEIR} FURTHER EXTENSION.*

\section{BY J. I NGHA M I K I N, Ese.,}

Pellow of the Royal College of Surgeons of England, and Lecturer on Anatomy and Physiology in the Leeds School of Medicine, etc.

Read before the Leeds Pbilosophical Society, March, 1851.

WITh regard to the progress of military hygiène I might also say a good deal ; butt here also I must forbear ; and not wishing to be invidious, will only allude to a few names in connexion with this honorable profession-this efficient upholder of our country's glory - this best preserver of peace, and check upon the tyranny of numbers. By congregating men together in armies disease has frequently broken out with great virulerice. But the cause of this has almost always been from some gross inattention or ignorance of the principles of hygiène. Ignorance of the nature of the soil on which an army is encamping - of the products of the country-of the diseases of the climate-the condition and nature of the food, have too often been deplorably extibited in the history of the campaigns of our army. But military hygiène is no longer disregarded by the medical officer or the general in command; and in the last war the army, under that efficient and indefatigable public servant-that honor to our profession, Sir James McGrigor, was a perfect model with respect to attention to preserving the health of the soldier. Choice of quarters-arrangement of uniform-security of, and attention to, the sick and wounded-attention to diet and the general physical condition and comfort of the soldier; nothing of any moment escaped the eagle eye of this experienced surgeon; and his immortal General, Wellington, knew well how to appreciate the services of such a man.

When Sir James McGrigor (vide Lancet's account) $†$ was appointed Director-General of the medical department of the army, he found it imperfect, the same as Sir Wm. Burnett had done the navy; no professional records of the state of health of the army, or its diseases, or their treatment, existing. No records, except those of a fiscal

- Continued from page 514.

+ See Biographical Sketch of Sir James McGrigor, in the Lancet, ane of the series now publishing. 